\title{
Abordagem multiprofissional quanto à promoção e prevenção de lesão por pressão em pacientes acamados em um hospital em Belém do Pará: um relato de experiência
}

Multiprofessional approach to the promotion and prevention of pressure injuries in bedridden patients in a hospital in Belém do Pará: na experience report

Enfoque multiprofesional para la promoción y prevención de lesiones por presión en pacientes encamados em um hospital de Belém do Pará: um informe de experiencia

Ana Karina Rodrigues Coelho ORCID: https://orcid.org/0000-0001-8504-689X Centro Universitário Fibra, Brasil E-mail: anna.karinna1999@gmail.com

Fábio Roberto de Sales Rodrigues Maia Filho ORCID: https://orcid.org/0000-0002-0729-1870 Universidade Federal do Pará E-mail: fabiorobertomaia@hotmail.com

Tamires Costa Franco ORCID: https://orcid.org/0000-0002-8978-4176

Universidade da Amazônia, Brasil

E-mail: tamiresfranco98@gmail.com

Barbara Maria Neves Mendonça Luz ORCID: https://orcid.org/0000-0001-7907-9337 Centro Universitário do Estado do Pará, Brasil

E-mail: barbaramendoncaluz@ outlook.com

Suely Patrícia Perdigão Abreu ORCID: https://orcid.org/0000-0001-9802-6943

Universidade da Amazônia, Brasil E-mail: suelypatricia8@gmail.com

Paulo Sergio Caetano de Carvalho ORCID: https://orcid.org/0000-0002-1958-9746 Universidade do Estado do Pará, Brasil E-mail: pscarvalhoo21@gmail.com João de Souza Mendonça ORDIC: https://orcid.org/0000-0002-2204-5010 Universidade da Amazônia, Brasil E-mail: joaosm.farmaco@gmail.com

Leidiane de Jesus da Costa Santos ORCID: https://orcid.org/0000-0002-9208-2319

Universidade da Amazônia, Brasil

E-mail: leidianesantos2023@gmail.com

Maria Eduarda Monteiro Pereira ORCID: https://orcid.org/0000-0002-3679-9120 Centro Universitário do Estado do Pará, Brasil

E-mail: mariamontpereira@outlook.com

Natasha Cristina Rangel Rodrigues ORCID: https://orcid.org/0000-0001-7441-9136 Universidade Federal do Pará, Brasil E-mail: natasharange194@ hotmail.com

Fernanda Maria Ribeiro Batista ORCID: https://orcid.org/0000-0001-9967-2381 Universidade Federal do Pará, Brasil E-mail: fehtista09@gmail.com

Amanda Almeida Vieira ORCID: https://orcid.org/0000-0003-2211-0115 Centro Universitário do Estado do Pará, Brasil E-mail: amandaav2000@gmail.com Jessica Luiza Lima Santos

ORCID: https://orcid.org/0000-0002-8180-0995 Universidade da Amazônia, Brasil E-mail: jessicaluiza79@gmail.com 


\author{
Manuele Figueira Printes \\ ORCID: https://orcid.org/0000-0003-3286-0497 \\ Universidade Norte do Paraná, Brasil \\ E-mail: figueiramanuele9@gmail.com \\ Vinicius Rodrigues dos Reis Amorim \\ ORCID: https://orcid.org/0000-0002-0873-9374 \\ Faculdade Mauricio de Nassau, Brasil \\ E-mail: viniciusamorim241@gmail.com
}

\begin{abstract}
Resumo
A lesão por pressão (LPP) é originada por uma interrupção sanguínea em uma determinada área do corpo, que se desenvolve devido a uma pressão por um longo período acamado em leitos de Unidade de Terapia Intensiva (UTI). A LPP diferentemente das demais alterações de pele, tem sido fonte de preocupação por representar um problema de saúde pública, levando a transtornos físicos, emocionais e influindo a morbidade e mortalidade. Trata-se de um estudo de caráter descritivo, do tipo relato de experiência, realizada em uma enfermaria de um hospital de referência em Belém no estado do Pará, durante as práticas de estagio supervisionado. Os pacientes que necessitam passar pela UTI, e que estão por indeterminados motivos acamados, acabam desenvolvendo LPP, devido a umidades, fricção, entre outros fatores. A assistência de enfermagem junto a equipe multiprofissional é crucial para que haja evolução de melhora na lesão, sem causar danos ao paciente, além de proporcionar a melhor aderência de tratamento. Portanto, há necessidade de repassar aos profissionais o que foi observado, para que prestem assistência multiprofissional de forma clara, para que não ocorra nenhuma intercorrência que pode ser causada pela LPP.
\end{abstract}

Palavras-chave: Assistência de enfermagem; Lesão por pressão; Saúde pública.

\begin{abstract}
:
Pressure injury (LPP) is caused by a blood interruption in a certain area of the body, which develops due to pressure for a long period of bed in Intensive Care Unit (ICU) beds. PPL, unlike other skin changes, has been a source of concern for representing a public health problem, leading to physical and emotional disorders and influencing morbidity and mortality. This is a descriptive study, of the experience report type, carried out in an infirmary of a reference hospital in Belém, Pará, during supervised internship practices. Patients who need to go to the ICU, and who are bedridden for undetermined reasons, end up developing PPL, due to humidity, friction, among other factors. Nursing care with the multidisciplinary team is crucial for the evolution of improvement in the lesion, without causing harm to the patient, in addition to providing the best treatment adherence. Therefore, there is a need to pass on to professionals what was observed, so that they provide multidisciplinary care in a clear way, so that no complications that may be caused by LPP occur.
\end{abstract}

Keywords: Nursing care; Pressure injury, Public health.

\title{
Resumen
}

La lesión por presión (LPP) es causada por una interrupción de la sangre en una determinada área del cuerpo, que se desarrolla debido a la presión durante un largo período de cama en las camas de la Unidad de Cuidados Intensivos (UCI). La PPL, a diferencia de otros cambios cutáneos, ha sido motivo de preocupación por representar un problema de salud pública, ocasionando trastornos físicos y emocionales e influyendo en la morbilidad y la mortalidad. Se trata de un estudio descriptivo, del tipo relato de experiencia, realizado en una enfermería de un hospital de referencia en Belém, Pará, durante prácticas supervisadas de internado. Los pacientes que necesitan acudir a la UCI, y que se encuentran postrados en cama por motivos indeterminados, acaban desarrollando LPP, por humedad, fricción, entre otros factores. La atención de enfermería con el equipo multidisciplinar es crucial para la evolución de la mejoría de la lesión, sin causar daño al paciente, además de brindar la mejor adherencia al tratamiento. Por tanto, existe la necesidad de transmitir a los profesionales lo observado, para que brinden una atención multidisciplinar de forma clara, para que no se produzcan complicaciones que puedan ser provocadas por la LPP.

Palabras clave: Cuidado de enfermera; Lesión por presión; Salud pública.

\section{Introdução}

A definição internacional de National Pressure Ulcer Advisory Panel (NPUAP) para lesão por pressão (LPP) é um dano localizado na pele ou em tecidos subjacentes, normalmente sobre uma proeminência óssea também relacionada ao uso de dispositivo medico ou outro artefato. A lesão ocorre como resultado de intensa e/ou prolongada pressão ou pressão combinada com cisalhamento e pode se apresentar em pele íntegra ou como úlcera aberta, e ser dolorosa. (NPUAP, 2016). 
O significado da palavra ferida tem como definição de perda da solução de continuidade da pele, e se isso acontecer em áreas consideradas prejudicadas, a exemplo: proeminência óssea, poderá evoluir rapidamente para a formação de uma lesão por pressão. (Neves \& Santos, 2013).

As LPP constituem um desafio para os serviços de saúde, pois sua ocorrência é considerada um indicador de qualidade da assistência de enfermagem prestada aos pacientes. Com isso, seu surgimento traz implicações tanto para quem a desenvolve, quanto para a instituição onde o paciente se encontra internado, pois, além de causar dor e desconforto pode retarda o processo de recuperação, e ainda aumentar as taxas de infecção, o que corrobora o prolongamento de internação. (Manganelli et al.,2019).

O paciente hospitalizado por ter sua condição fisiológica comprometida pela patologia apresentada, necessita de cuidados de aspectos físicos, psicológicos e sociais, ou seja, de um cuidado integral. (Almeida et al., 2019).

O cuidado voltado as pessoas com LPP deve ocorrer na alta e média complexidade, bem como na Atenção Primária à Saúde (APS), sob os cuidados da Equipe de Saúde da Família (ESF), o que remete os esforços para o estabelecimento de diretrizes que norteiem a pratica da prevenção, sendo relevante o avanço para ações de promoção de saúde. (Soares \& Heidemann, 2019).

A Enfermagem, por se tratar da maior força de trabalho em saúde no Brasil, remete a necessidade de uma relação direta da categoria com as estratégias de segurança do paciente e a prevenção de erros. A realização de notificação de eventos adversos é necessária, pois contribui para o acompanhamento e controle das ocorrências e para a elaboração de medidas preventivas mais eficazes. (Pachá et al., 2018).

Logo, quando o processo de prevenção e cuidados são empregados adequadamente e mesmo assim as lesões se instalam, todos os envolvidos, iniciam uma peregrinação em busca de soluções que causam significante impacto social e econômico devido à natureza recorrente do longo tempo entre a abertura da feriada e sua cicatrização. Para isso, é importante saber como prevenir, quais as fases e risco da formação da lesão por pressão, e classificar o seu grau de gravidade, assim como identificar indivíduos em risco que necessitem de prevenção e os fatores desencadeantes. (Lucri \& Costa, 2021).

De acordo com o National Pressure Ulcer Advisory Panel (NPUAP), as lesões por pressão podem ser classificadas em quatro estágios, sendo eles: Estágio I -pele íntegra com eritema que não embranquece; estágio II - perda da pele em sua espessura parcial com exposição da derme; estágio III - perda da pele em sua espessura total; e estágio IV - perda da pele em sua espessura total e perda tissular. A partir deste nível, que a lesão fica mais profunda, ela fica não classificável.

As lesões por pressão, mesmo sendo um evento prejudicial que na maioria das vezes pode ser evitado. Existem algumas situações que mesmo com medidas preventivas, as condições fisiológicas do paciente tornam as lesões inevitáveis. Pacientes com sensibilidade diminuída, idade avançada, imobilidade prolongada ou restrita ao leito são mais susceptíveis à lesão por pressão, que acometem frequentemente nas regiões occipital, escapular, cotovelo, sacral, ísquio, crista ilíaca, joelho, maléolo e calcâneo. (Araújo et al., 2019).

Os altos índices de lesões por pressão, estão relacionados a uma assistência precária, enquanto que os baixos índices representam que a assistência à saúde está superando os fatores intrínsecos e extrínsecos passiveis de prevenção que são responsáveis pelo desencadeamento das LPP. (Lima et al., 2017).

Apesar da ocorrência de LPP ser considerada um evento adverso que pode, na maioria das vezes ser evitado, existem algumas ocasiões que mesmo utilizando medidas de prevenção, as condições fisiológicas do paciente tornam as lesões inevitáveis. Outros fatores que aumentam o risco de desenvolver LP englobam doenças degenerativas, tolerância tecidual reduzida (pele frágil), incontinência urinária ou intestinal e desnutrição ou obesidade. Nestes casos, é crucial a implementação de cuidados adequados, o que instiga o enfermeiro a buscar capacitação para o reconhecimento do problema e para novas intervenções. (Mazzo et al., 2018). 
Diante da vulnerabilidade de pacientes com lesão por pressão ao desenvolvimento de outras patologias e infecções, torna-se importante as discussões e implementação de segurança para o paciente como norteador do cuidado, tanto da equipe de enfermagem, quanto da equipe multiprofissional que se empenha no tratamento do paciente. (Zimmermann et al., 2018).

\section{Metodologia}

Este estudo caracteriza-se como descritivo, do tipo relato de experiência, realizado em uma enfermaria de um hospital de referência de Belém no estado do Pará, no período de agosto de 2021, durantes as práticas de estágio supervisionado II.

Durante as práticas de enfermagem em uma enfermaria notou-se a importância de uma assistência em enfermagem de qualidade, quanto aos cuidados nas lesões por pressão, onde foi possível acompanhar e realizar os curativos em lesões extensas e profundas, e se observou a fragilidade de cada paciente em relação as lesões, que causavam dor e desconforto em pacientes acamados.

Com base na Escala de Braden como instrumento de prevenção que permite estratificar o risco de desenvolvimento de lesão por pressão, através de suas aplicabilidade, fornece ao enfermeiro elaborar um planejamento preventivo e identificar riscos de forma individualizada. (Silva et al., 2019).

A Escala de Braden, é uma ferramenta, de escala de score, com a pontuação máxima de 18 e pontuação mínima de 6 , sendo que quanto menor o score, maior o risco de desenvolvimento de lesão por pressão. Através da aplicação da Escala de Braden avaliam-se seis fatores que influenciam o risco de desenvolvimento lesão por pressão, sendo eles: percepção sensorial, umidade, mobilidade, estado nutricional, nível de atividade e fricção e forças de deslizamento. A partir desse registro, enfermeiros conseguem planejar medidas preventivas a serem aplicadas e promover um tratamento mais eficaz (CARVALHO, 2016).

O fundamento teórico decorreu pela busca bibliográfica em bases de dados científicas digitais, sendo elas: Scientific Electronic Library Online (SCIELO) e Biblioteca Virtual em Saúde (BVS), com a utilização de palavras-chaves colhidas nos Descritores em Ciências da Saúde (DeCS), sendo elas: "Assistência de enfermagem"; " Lesão por pressão"; " Saúde Pública".

Esta etapa visou fundamentar as metas que seriam desenvolvidas, bem como promover maior conhecimento científico e relevância ao estudo.

\section{Resultados}

A enfermagem é uma ciência que tem como objeto o cuidado. Nesta perspectiva o enfoque preventivo, assim como o de promoção da saúde, deve nortear a prática assistencial, na busca por um menor índice da lesão por pressão. (Soares \& Heidemann, 2019).

Com base na experiência vivida, notou que alguns dos pacientes acamados, estavam desmotivados durante o dia a dia, por conta da lesão principalmente na área lombar e sacral, que causam certo desconforto no paciente.

Durante a limpeza e curativos das lesões, havia interação entre profissional e paciente, para que o mesmo tivesse conhecimento do procedimento que estava sendo feito. Era utilizado hidrogel, gazes e fita micropore para realizar o fechamento do curativo. Sendo o hidrogel, com efeito cicatrizante, uma pomada bastante utilizada em meios hospitalares para tratamento de feridas causadas por LPP.

Alguns dos pacientes, pode-se observar que estavam desanimados, pois a ferida já estava por um longo período na região sacral e lombar, e causando novas lesões principalmente no calcâneo por conta do cisalhamento. Com base na 
assistência de enfermagem prestada, realizou-se de forma higiênica a limpeza das lesões, bem como o fechamento dos curativos.

O uso de Equipamento de Proteção Individuais (EPI's), é de extrema importância no momento da realização das feridas, para que não haja contaminação do meio externo para o meio interno, visto que pacientes acamados e com lesões por pressão estão mais suscetíveis a riscos de contaminação por bactérias, entre outros microrganismos.

Foi utilizado como prevenção e promoção de saúde, a mudança de decúbito do paciente, hidratação corporal, nutrição adequada, entre outros fatores, são indispensáveis na evolução do paciente, para que ele se sinta acolhido e motivado a buscar forças para o tratamento, visto que a lesão por pressão é uma causa subjacente. Sendo que uma boa parte de pacientes acamados por LPP, são hipertensos(as), diabéticos(as), entre outras comorbidades, o que pode interferir no tratamento da lesão.

Durante as realizações de limpezas das lesões, foi repassado para o paciente e acompanhante sobre a caminhada pelo próprio quarto onde estava internado, se o mesmo não tivesse sentindo dor ou desconforto, afim de que se evite novas lesões em outras partes do corpo.

Pelo tempo indeterminado do paciente acamado, e pelo desconforto que a lesão causa, a importância da equipe multiprofissional se torna de suma importância no estado geral do paciente, como forma de assistência hospitalar, para que gerem futuramente bons resultados, sem nenhuma intercorrência.

Com o apoio de médicos, fisioterapeutas, nutricionista e de toda a equipe, foi possível estabelecer metas para cada paciente com LPP, para que pudéssemos avançar na melhora daquela lesão, bem como medicações para alivio das dores, alimentação adequada que é fundamental para uma boa resistência do organismo do paciente, assim como sessões de fisioterapia, visto que alguns pacientes não conseguiam se locomover devido a dor causada pela lesão.

Leite (2017) complementa que para um cuidado efetivo, a prescrição de enfermagem deve contemplar estratégias para efetivação de um plano minucioso a ser aplicado ao paciente acamado que desenvolve, ou poderá desenvolver lesão por pressão. O papel do profissional de enfermagem juntamente com toda equipe multiprofissional tem sua responsabilidade na prevenção, tratamento e cuidado com as feridas causadas por LPP, e da necessidade de toda equipe estar ciente dos avanços recentes e suas implicações, para que haja um atendimento de qualidade e efetivo.

\section{Discussão}

A identificação dos fatores individuais de risco é útil para realização de uma Sistematização da Assistência de Enfermagem (SAE) no cuidado e na terapêutica. Torna-se então essa assistência uma ação eficaz na prevenção de lesão por pressão, oferecendo ao paciente aquilo que é a essência da profissão: cuidar do outro quando este não consegue fazê-lo ou precisa de auxílio como orientação ou supervisão. (Silva \& Silva, 2018).

A atuação do enfermeiro na gerência do cuidado é um dos principais eixos na atuação profissional da categoria, compreendendo a articulação entre as dimensões assistenciais e gerenciais na execução das atividades (Costa et al., 2020).

A importância deste assunto refere-se na importância da assistência de enfermagem para reconstituição da pele, causada pela lesão por pressão em pacientes acamados, que ocasionalmente passaram por longo tempo em uma Unidade de Terapia Intensiva (UTI) ou estão um longo tempo em enfermarias acamados.

Cabe a equipe multiprofissional, o acompanhamento individual de cada paciente, a fim de que haja uma melhora no estado físico, psicológico e social, visto que em algumas circunstâncias o próprio paciente se sente constrangido pelo estado no qual a lesão se encontra.

A conversa com o paciente, e uma escuta durante o procedimento de limpeza, pode trazer benefícios, tanto para o paciente, quanto para a equipe, pois em alguns momentos durante a visita de enfermagem, alguns pacientes não relatam o que sentem, o que é possível observar ao contrário durante as limpezas de feridas, como queixas de prurido no local da lesão. 
A mudança de decúbito, troca de colchoes e roupas de cama em ambiente hospitalar, possuem ligação direta na predisposição de LPP, e devem ser levadas em consideração em pacientes acamados, possuindo um papel fundamental para o tratamento das lesões, e proporcionar bem estar e higiene aos pacientes.

É fundamental o conhecimento cientifico teórico dos profissionais de saúde, afim de que possibilite uma melhora no estado geral do paciente, favorecendo um cuidado humanizado e amplo.

O modelo de ensino tradicional vem sendo aos poucos substituído por novas tendências e implicações as quais apontam para a necessidade da formação de um profissional crítico-reflexivo, capaz de transverter a realidade social do seu cotidiano em benefícios acessíveis para a comunidade ao qual é inserido, tendo em vista proveitos coletivos que influenciarão diretamente na qualidade assistencial futura (Cunha et al., 2021).

\section{Conclusão}

Os profissionais de enfermagem tem um papel fundamental na melhoria desses pacientes acometidos por conta da lesão por pressão, pode-se perceber que uma boa assistência de enfermagem, remete a melhoria do paciente de forma humanizada e delicada.

Para mais, é importante ratificar neste trabalho que os profissionais de enfermagem, junto a equipe multiprofissional, atuem na prevenção, promoção e tratamento dos pacientes. Em síntese, espera-se que este trabalho possa contribuir com o desenvolvimento cientifico, e na elaboração de estratégias para acadêmicos e profissionais que já atuam na área, sobre a importância da promoção e prevenção de saúde, para que não se utilize meios invasivos em pacientes que já estão consideravelmente frágeis. A fim de proporcionar um cuidado humanizado, sem intervenções cirúrgicas, para que o paciente evolua no seu quadro de forma positiva e sem intercorrência.

\section{Referências}

Almeida, F., Costa, M. M. S. d., Ribeiro, E. E. S., Santos, D. C. O., Silva, N. D. A., Silva, R. E., Saraiva, K. P. \& Pereira, P. C. B. (2019). Assistência de enfermagem na prevenção da lesão por pressão: uma revisão integrativa.

Araújo, J. F., Mello, E. C. A. \& Santos, E. A. (2019). Lesão por pressão em unidade de terapia intensiva: abordando a assistência de enfermagem. Temas em Saúde Fesvip. 130 a 142.

Cunha, A. G., Silva, A. F. L. d., Mendes, A. P. d. S., Oliveira, A. K. C. d., Braga, B. S. C., Silva, B. C. M. d., Portilho, D. C., Portilho, D. C., Nascimento, J. L. M., Silva, J. M. L. d., Soeiro, J. d. S., Ferreira, L. F., Carvalho, M. A., Ferreira, P. d. S., Carvalho, P. H. C. d., \& Silva, R. R. d. (2021). Uso de metodologias ativas na promoção do autocuidado e adesão terapêutica com usuários de um centro de atenção psicossocial. Research, Society and Development, $10(1), 1-7$.

Leite, G. R. (2017) Protocolo de cirurgias seguras numa região neotropical no Brasil Central. (Pós-Graduação), Universidade Federal de Goiás. UFG, Goiás, Go. Brasil.

Lima, P. R., Damacena, D. E. L., Neves, V. L. S., Campos, R. B. N., Silva, F. A. A. d., \& Bezerra, S. M. G. (2017). Ocorrência De Lesão Por Pressão Em Pacientes Hospitalizados: Uma Revisão Integrativa. 1-15

Lucri, M. J. d. S., \& Costa, M. d. O. (2021). A assistência da enfermagem nas lesões por pressão em pacientes acamados. 1-16.

Manganelli, R. R., Kirchhof, R. S., Pieszak, G. M., \& Dornelles, C. d. S. (2019). Intervenções de enfermeiros na prevenção de lesão por pressão em uma unidade de terapia intensiva. 2-10.

Mazzo, A., Miranda, F. B. G., Meska, M. H. G., Bianchini, A., Bernardes, R. M., \& Junior, G. A. P. J. (2018). Ensino de prevenção e tratamento de lesão por pressão utilizando simulação.

National Pressure Ulcer Advisory Panel (NPUAP) (2016).

Neves, R. C., Santos, C. O. (2013). Escalas utilizadas para prevenir úlceras por pressão em pacientes críticos. Revista Enfermagem Contemporânea. 2(1),1931.

Pachá, H. H. P., Faria, J. I. L., Oliveira, K. A. d., \& Beccaria, L. M. (2018). Lesão por Pressão em Unidade de Terapia Intensiva: estudo de caso-controle. 1-8.

Silva, A. L. M. d., Rached, C. D. A., \& Liberal, M. M. C. D. (2019). A Utilização Da Escala De Braden Como Instrumento Preditivo Para Prevenção De Lesão Por Pressão. 
Research, Society and Development, v. 10, n. 12, e18101219893, 2021

(CC BY 4.0) | ISSN 2525-3409 | DOI: http://dx.doi.org/10.33448/rsd-v10i12.19893

Silva, M. S. d., \& Silva, C. d. C. (2018). Assistências de enfermagem ao paciente com úlcera por pressão na unidade de terapia intensiva: revisão integrativa

Soares, C. F., \& Heidemann, I. T. S. B. (2019). Promoção Da Saúde E Prevenção Da Lesão Por Pressão: Expectativas Do Enfermeiro Da Atenção Primária. 19.

Zimmermann, G. S., Cremasco, M. F, Zanei, S. S. V., Takahashi, S. M., Cohrs, C. R., \& Whitaker, I. Y. (2018). Predição de risco de lesão por pressão em pacientes de unidade de terapia intensiva: revisão integrativa. Texto \& contexto enferm, 27(3), e3250017 